\title{
A first case report of clinical response to targeted therapy in a patient with primary myoepithelial carcinoma of the lung harboring EGFR exon 19 deletion
}

Xiaohong Xie ${ }^{1}$, Xinqing Lin ${ }^{1}$, Ming Liư ${ }^{1}$, Yinyin Qin ${ }^{1}$, Ming Ouyang ${ }^{1}$, Shiyue Li ${ }^{1}$, Yingying $\mathrm{Gu}^{2}$, Shuyin Chen ${ }^{3}$, Jianxing Xiang ${ }^{3}$ and Chengzhi Zhou ${ }^{1 *}$ (D)

\begin{abstract}
Background: Primary myoepithelial carcinoma of the lung is a rare subtype in lung cancer. Comprehensive molecular profiling of myoepithelial carcinoma of the lung is absent, neither was clinical evidence of targeted therapy available for this disease. Therefore, the optimal treatment regimen of this tumor needs to be established.

Case presentation: Here we present a case of a 68-year-old patient with stage IVB primary myoepithelial carcinoma of the lung who harbored EGFR exon 19 deletion and KRAS mutation and underwent icotinib targeted therapy, achieving partial response (PR) with progression free survival (PFS) of 3 months.

Conclusion: To our knowledge, this study describes the first documented case of primary myoepithelial carcinoma lung cancer patient harboring EGFR exon 19 deletion and KRAS mutation, and showed clinical efficacy of epidermal growth factor receptor tyrosine kinase inhibitors (EGFR-TKI) treatment in this patient.
\end{abstract}

Keywords: Lung, Myoepithelial carcinoma, Salivary gland type tumor, EGFR 19del, Icotinib

\section{Introduction}

Myoepithelial Carcinoma of the lung is a rare subset of primary salivary-type tumors, accounting for less than $1 \%$ in all lung tumors [1]. It was first described in 1975 by Stromeyer et al., and modified by Dardick et al., with the histopathologic guidelines in 1995 [2]. It seems that due to the lack of sufficient knowledge and diagnostic criteria for this tumor in the past, the incident rate of this tumor may be underestimated [3].

\footnotetext{
* Correspondence: zcz2019asco@163.com

'Department of Pulmonary and Critical Care Medicine, The First Affiliated Hospital of Guangzhou Medical University, Guangzhou Institute of Respiratory Health, State Key Laboratory of Respiratory Disease, National Clinical Research Center for Respiratory Disease, 151 Yanjiang Road, Guangzhou 510120, China

Full list of author information is available at the end of the article
}

Myoepithelial carcinoma usually occurs in middleaged adults, the average age at diagnosis is around 55 years old. Although it mainly occurs in the parotid gland, it can also be found in primary sites other than the salivary glands, such as lung, skin and soft tissue [4-6]. The molecular profiling of these tumors has not been systematically studied. EGFR mutation is very common in lung adenocarcinoma, but it has not been reported in this tumor owing to the rarity of the disease and lack of attempts to characterize it molecularly. Therefore, it is unknown whether targeted therapies such as EGFR-TKI could achieve similar response in primary myoepithelial carcinoma as in lung adenocarcinoma. 


\section{Case report}

A 68-year-old man presented to our institution for the treatment with cough and chest pain which already last for 4 months. He had a medical history of chronic gastritis for 3 years, and he was a smoker (30 cigarettes/day) for 40 years. He denied other symptoms like nausea, fever, vomiting or shortness of breath. Electrocardiography shows $\mathrm{T}$ wave changes and echocardiography showed moderate tricuspid regurgitation. The left ventricular diastolic pressure decreased. Routine laboratory examinations found carcinoembryonic antigen (CEA) at $4.7 \mathrm{ng} / \mathrm{mL}$ (normal
A.

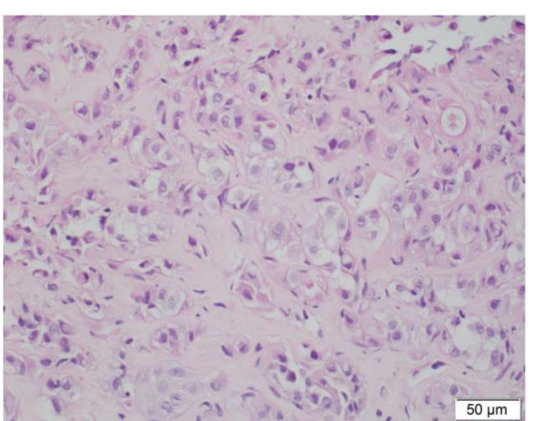

C.

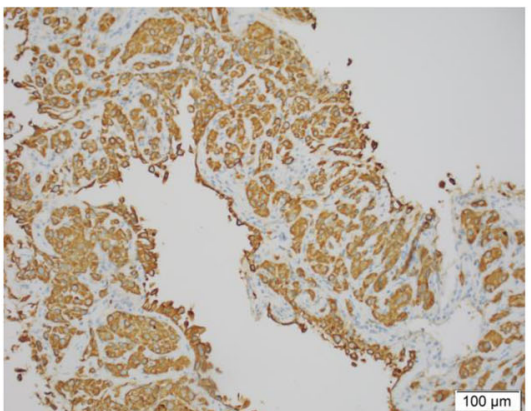

D.

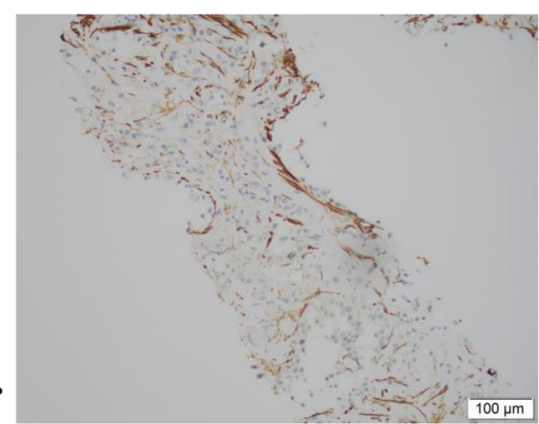

B.

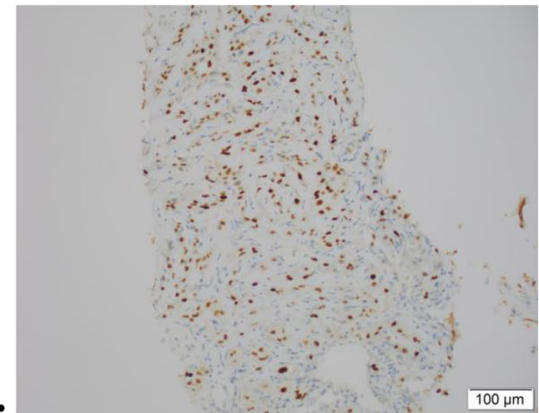

E.

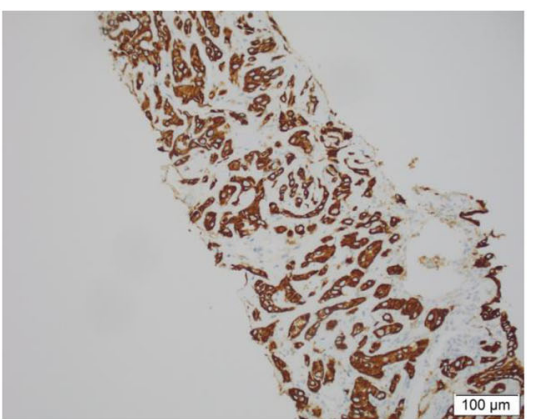

F.

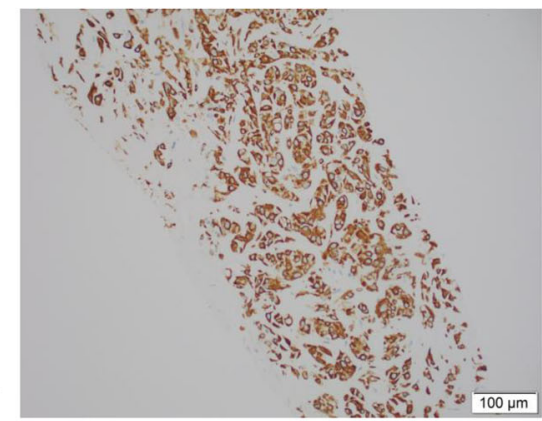

G.

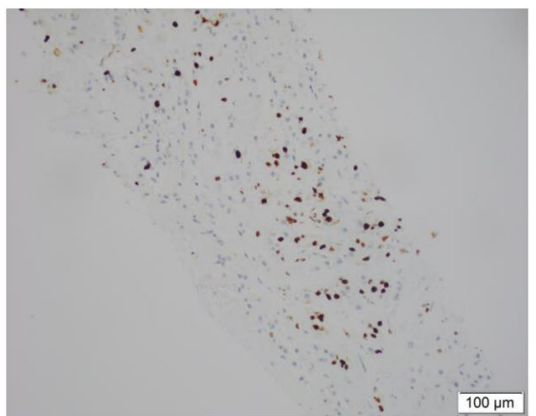

H.

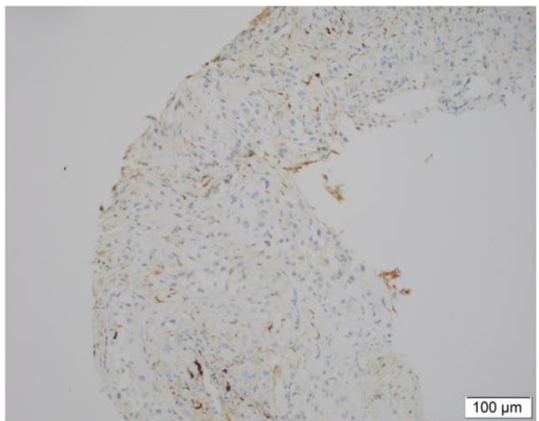

Fig. 1 Histologic features of primay myoepithelial carcinoma of the lung. a Hematoxylin-eosin (HE) stain (magnification X400): The cancer cells are arranged like small nests with many hyaline substances in the stroma; b-h. Immunohistochemical staining of Calponin (b), CK (c), P63 (d), CK7 (e), CK5/6 (f), Ki67 (g), S-100 (h) (magnification X200) 
range, $<5 \mathrm{ng} / \mathrm{mL}$ ) within normal limits, but increased level of Prostate-specific antigen (PSA) $(5.1 \mathrm{ng} / \mathrm{mL}$; normal range, $<4.4 \mathrm{ng} / \mathrm{mL}$ ), cancer antigen 125 (CA125) $(41.7 \mathrm{U} / \mathrm{mL}$; normal range, $<35 \mathrm{U} / \mathrm{mL})$, and carbohydrate antigen 19-9 (CA19-9) (53.4.1 U/mL; normal range, $<27 \mathrm{U} / \mathrm{mL}$ ) in the serum. Furthermore, the fiberoptic bronchoscopic biopsy sample was fixed with $10 \%$ neutral formalin, embedded in paraffin, routinely prepared, stained with hematoxylin-eosin, and observed under light microscope. Streptavidinperosidase method was used for immunohistochemistry. The Immunohistochemical staining results showed that the tumor cells were positive for $\mathrm{CK}(+)$, P63(+), CK7(+), CK5/6(+), Ki67(+), S-100(+), Calpo$\operatorname{nin}(+)$, while negative for P40, NapsinA, TTF1, WT-1 and CR (Fig. 1).

Based on the radiopathological result, he was diagnosed with stage IVB myoepithelial carcinoma accompanied by metastases in the left pulmonary hilum, mediastinal lymph nodes, and cervical vertebra. Besides, there was no measurable tumor observed from PET-CT along his salivary gland. His Eastern Cooperative Oncology Group (ECOG) performance status was 1, and his vital signs were normal.
Furthermore, the biopsy samples were tested by targeted next generation sequencing (NGS) with a 168gene cancer panel (Burning Rock Biotech, Guangzhou, Guangdong, China). The NGS sequencing results revealed EGFR exon 19 deletion (p.E746_A750del; $0.04 \%$ ) and KRAS mutation (p.G12C; 0.15\%) from plasma sample, and only the KRAS mutation (p.G12C; 53.18\%) from needle biopsy tissue sample of the lung (Fig. 2). Subsequently, he was treated with EGFR-TKI icotinib at $125 \mathrm{mg}$ orally thrice daily from July 25th 2019. After 40 days, the patient came back for reexamination of chest CT. The radiography showed a significant reduction in his primary lung lesion $(6.2 \times 4.7 \mathrm{~cm}$ vs $4.2 \times 3.5 \mathrm{~cm})$. There was significant improvement in clinical symptoms including chest pain. He achieved partial response (PR) under the criteria of Response Evaluation Criteria In Solid Tumors (RECIST) 1.1 (Fig. 3). Unfortunately, the disease progressed after 2.5 months of EGFR-TKI treatment. The magnetic resonance imaging (MRI) revealed disseminated bone metastases and brain atrophy. On Oct 31st 2019, the patient passed away due to multiple organ failures with an overall survival (OS) of 3 months.

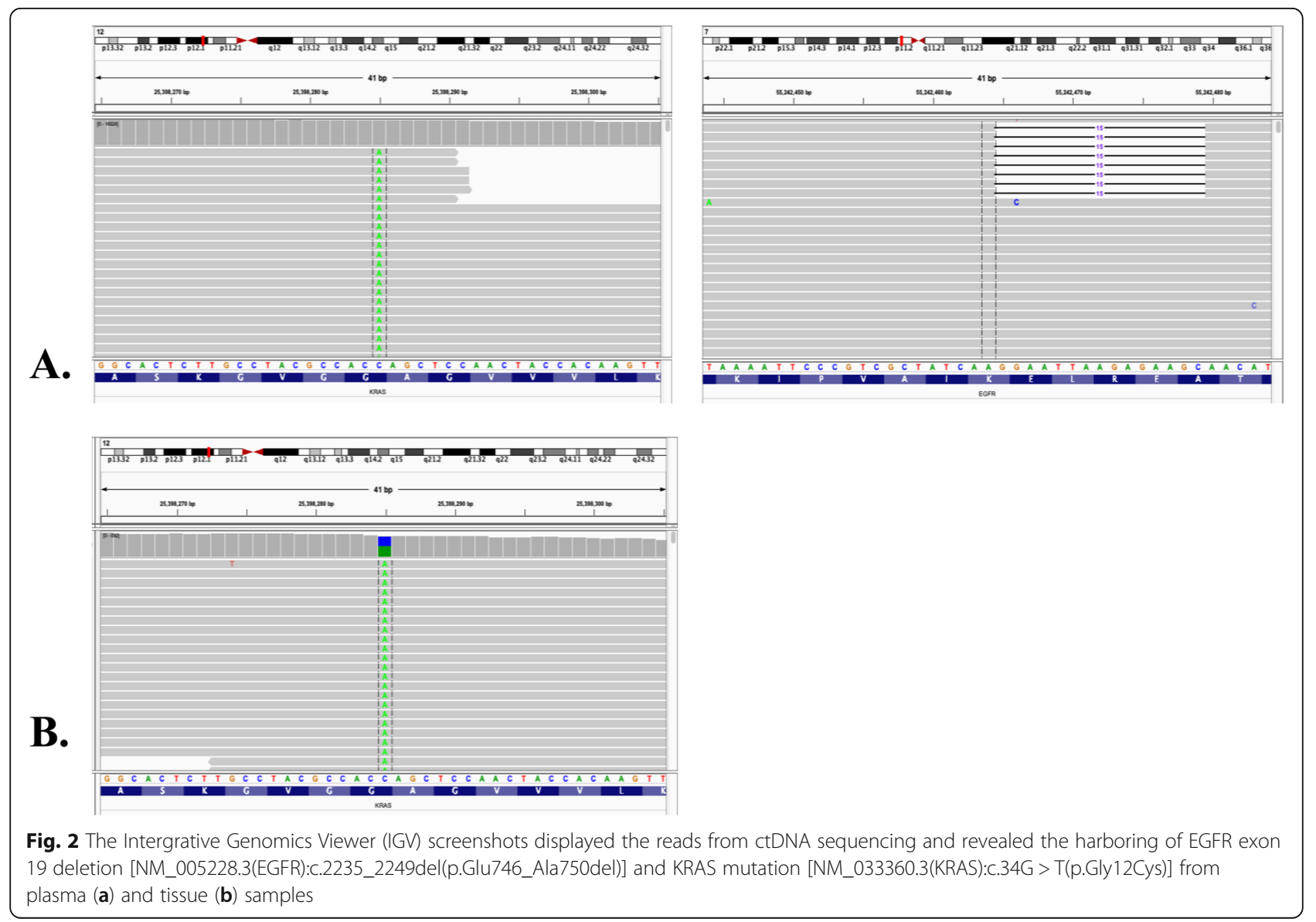




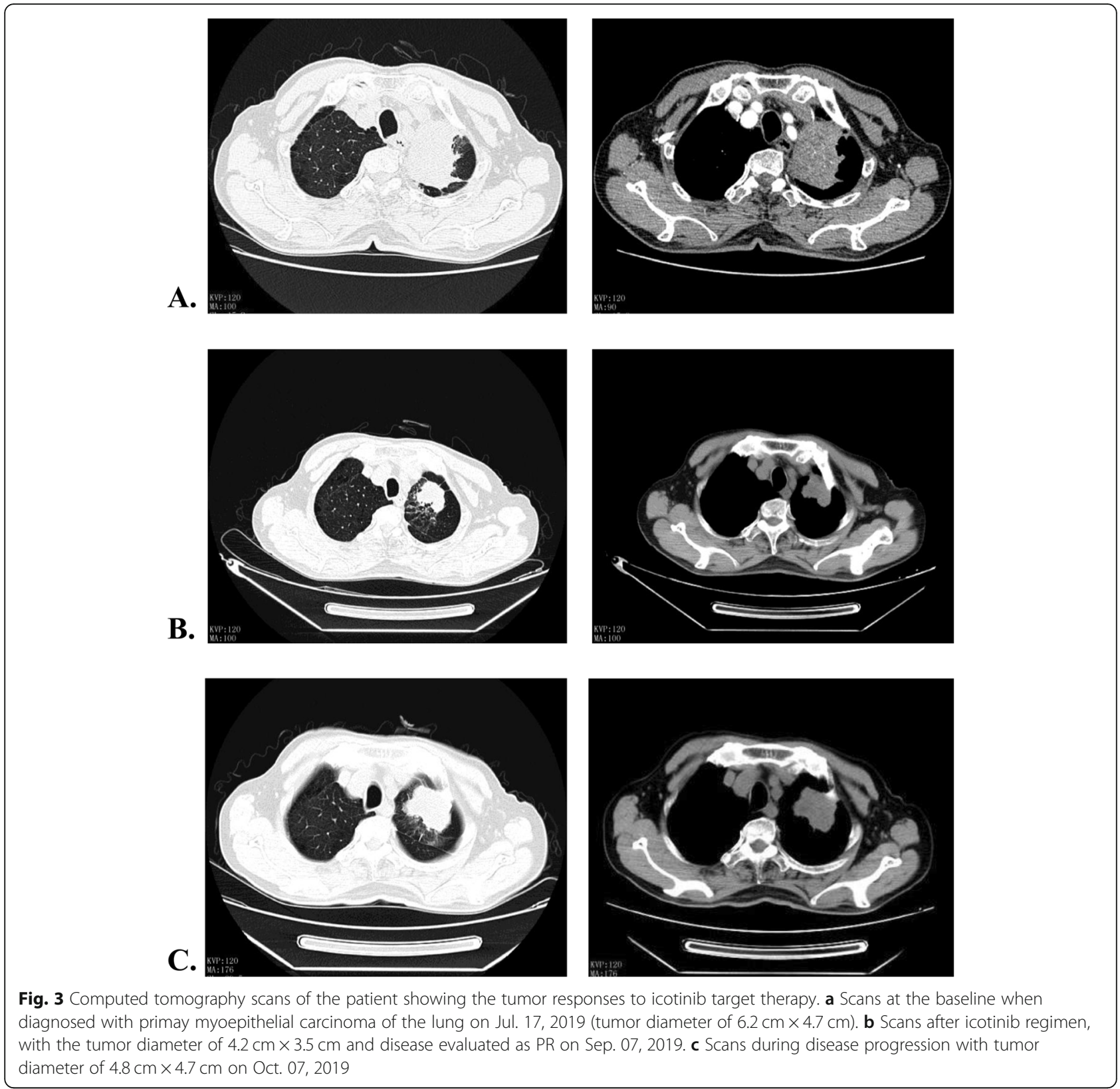

\section{Discussion}

The most common practice to manage localized myoepithelial carcinoma is by performing surgical excision with or without adjuvant radiation therapy [7]. However, myoepithelial carcinoma has a high tendency of local recurrences and metastases that warrants close clinical follow-up. After developing into advanced disease, systemic therapy plays an essential role in prolonging survival. Although chemotherapy was the most frequently used systemic therapy, some studies showed that this tumor is not sensitive to chemotherapy [8]. And there is limited evidence of targeted therapy and immunotherapy. Our case is unique because we presented the first clinical evidence of EGFR-TKI targeted therapy for myoepithelial carcinoma.

EGFR mutation is very common in lung cancer patients. But pulmonary myoepithelial carcinoma is a rare subtype of lung carcinoma; there is no previous report of myoepithelial carcinoma patient harboring EGFR mutation. To the best of our knowledge, this is the first time that a myoepithelial carcinoma patient was reported to carry EGFR mutation (exon 19 deletion) and achieve PR after EGFR-TKI therapy.

Previous studies in lung cancer suggested that KRAS mutation is a poor prognostic marker for 
response to EGFR TKI treatment [9-11]. Especially the KRAS subtype of G12C mutation showed significant shorter PFS and OS than other KRAS mutation types [12]. Taken a glance in the BENEFIT study, the lung adenocarcinoma patients with concurrent mutations in EGFR and other oncogenes, including KRAS, had significantly shorter PFS than those with EGFR mutations only (13.2 months VS 4.7 months, HR 2.66, 95\% CI 1.58-4.49; $p=0.0003$ ) [11]. Therefore, the relatively short PFS time in our myoepithelial carcinoma patient may be due to the accompanying gene aberrances. Also, clonal heterogeneity might explain this relatively short PFS outcome. From the molecular tests, EGFR mutation was only found in liquid biopsy with very low concentration. It indicated that EGFR might not constitute the major clone of the tumor, and rather the subclone which was missed out by needle biopsy. Instead, KRAS was considered as the dominant driver mutation in this case, which might explain the reduced efficacy achieved by EGFR targeted therapy.

In brief, this case provides evidence of the potential use of targeted therapy in primary myoepithelial carcinoma of the lung. In addition, it highlights the importance of NGS analysis with the baseline biopsy sample, which may aid in the determination and prognostic prediction of therapeutic choices. We believe that the molecular profiling of myoepithelial carcinoma warrants further investigations, which could help determine whether targeted therapy should be included as a routine therapeutic option in myoepithelial carcinoma.

\section{Conclusions}

In conclusion, we presented a case report demonstrating clinical evidence of the efficacy of icotinib in a patient with advanced myoepithelial carcinoma of the lung who harbored EGFR exon 19 deletion. We also provided evidence that molecular profiling and targeted therapy might be potential critical implications in this rare type of disease.

\section{Abbreviations \\ CEA: Carcinoembryonic antigen; PFS: Progression-free survival; TKI: Tyrosine kinase inhibitor; PR: Partial response; ECOG: Eastern Cooperative Oncology Group; RECIST: Response evaluation criteria in solid tumors; NGS: Next- generation sequencing; OS: Overall survival}

\section{Acknowledgments}

We owe thanks to the patients and his family. We also thank the stuff at from Burning Rock Biotech who worked on these case.

\section{Authors' contributions}

$\mathrm{XH}$ Xie acquired data. SY Chen drafted the manuscript under the supervision of $\mathrm{XH}$ Xie and $\mathrm{CZ}$ Zhou. All the authors have given final approval of the version to be published.

\section{Funding}

This work is supported by grants from the State Key Laboratory of Respiratory Disease- The Independent project [grant number: SKLRD-QN201720]; State Key Laboratory of Respiratory Disease- The open project [grant number: SKLRD-OP-2018011; and Guangdong High Level University Clinical Cultivation Project [grant number: 2017-21,020]; Wu Jieping Medical Foundation's special funding fund for clinical research (No.320.6750.18125).

\section{Availability of data and materials}

Is available upon request from the corresponding author.

\section{Ethics approval and consent to participate}

This study was approved by the Institutional Review Board at the the First Affiliated Hospital of Guangzhou Medical University. The patient provided informed consent and gave permission to this study.

\section{Consent for publication}

Not applicable.

\section{Competing interests}

S.C. and J.X. are employees of Burning Rock Biotech. The other authors declare no potential conflicts of interest.

\section{Author details}

${ }^{1}$ Department of Pulmonary and Critical Care Medicine, The First Affiliated Hospital of Guangzhou Medical University, Guangzhou Institute of Respiratory Health, State Key Laboratory of Respiratory Disease, National Clinical Research Center for Respiratory Disease, 151 Yanjiang Road, Guangzhou 510120, China. ²Department of Respiratory Pathology, The First Affiliated Hospital of Guangzhou Medical University, Guangzhou Institute of Respiratory Health, State Key Laboratory of Respiratory Disease, National Clinical Research Center for Respiratory Disease, Guangzhou 510120, China. ${ }^{3}$ Burning Rock Biotech, Guangzhou 510300, China.

Received: 9 April 2020 Accepted: 27 May 2020

Published online: 06 June 2020

\section{References}

1. Song DH, Choi $\mathrm{H}, \mathrm{Ha}$ SY, et al. Epithelial-myoepthelial carcinoma of the tracheobronchial tree: the prognostic role of myoepithelial cells. Lung Cancer. 2014;83(3):416-9.

2. Dardick I. Myoepithelioma: definitions and diagnostic criteria. Ultrastruct Pathol. 1995;19(5):335-45.

3. Savera AT, Sloman A, Huvos AG, Klimstra DS. Myoepithelial carcinoma of the salivary glands: a clinicopathologic study of 25 patients. Am J Surg Pathol. 2000;24(6):761-74

4. Dhawan A, Shenoy A, Sriprakash D. Myoepithelial carcinoma of the nasopharynx: case report of a rare entity. Natl J Maxillofac Surg. 2011;2(2): 207-9.

5. Noronha V, Cooper DL, Higgins SA, Murren JR, Kluger HM. Metastatic myoepithelial carcinoma of the vulva treated with carboplatin and paclitaxel. Lancet Oncol. 2006;7(3):270-1.

6. Jo VY, Fletcher CD. Myoepithelial neoplasms of soft tissue: an updated review of the clinicopathologic, immunophenotypic, and genetic features. Head Neck Pathol. 2015;9(1):32-8.

7. Chamberlain F, Cojocaru E, Scaranti M, et al. Adult soft tissue myoepithelial carcinoma: treatment outcomes and efficacy of chemotherapy. Med Oncol. 2019;37(2):13.

8. Miura K, Harada H, Aiba S, Tsutsui Y. Myoepithelial carcinoma of the lung arising from bronchial submucosa. Am J Surg Pathol. 2000;24(9):1300-4.

9. Linardou $H$, Dahabreh IJ, Kanaloupiti D, et al. Assessment of somatic k-RAS mutations as a mechanism associated with resistance to EGFR-targeted agents: a systematic review and meta-analysis of studies in advanced nonsmall-cell lung cancer and metastatic colorectal cancer. Lancet Oncol. 2008; 9(10):962-72.

10. Ludovini V, Bianconi F, Pistola $L$, et al. Phosphoinositide-3-kinase catalytic alpha and KRAS mutations are important predictors of resistance to therapy with epidermal growth factor receptor tyrosine kinase inhibitors in patients with advanced non-small cell lung cancer. J Thorac Oncol. 2011;6(4):707-15.

11. Wang Z, Cheng Y, An T, et al. Detection of EGFR mutations in plasma circulating tumour DNA as a selection criterion for first-line gefitinib 
treatment in patients with advanced lung adenocarcinoma (BENEFIT): a phase 2, single-arm, multicentre clinical trial. Lancet Respir Med. 2018;6(9): 681-90.

12. Fiala $\mathrm{O}$, Pesek $M$, Finek J, Benesova L Belsanova B, Minarik M. The dominant role of G12C over other KRAS mutation types in the negative prediction of efficacy of epidermal growth factor receptor tyrosine kinase inhibitors in non-small cell lung cancer. Cancer Genet. 2013;206(1-2):26-31.

\section{Publisher's Note}

Springer Nature remains neutral with regard to jurisdictional claims in published maps and institutional affiliations.

Ready to submit your research? Choose BMC and benefit from:

- fast, convenient online submission

- thorough peer review by experienced researchers in your field

- rapid publication on acceptance

- support for research data, including large and complex data types

- gold Open Access which fosters wider collaboration and increased citations

- maximum visibility for your research: over $100 \mathrm{M}$ website views per year

At $B M C$, research is always in progress.

Learn more biomedcentral.com/submissions 